\title{
IncRNA XIST regulates proliferation and migration of hepatocellular carcinoma cells by acting as miR-497-5p molecular sponge and targeting PDCD4
}

Yixi Zhang ${ }^{1,2,3 \dagger}$, Zebin Zhu ${ }^{1,2,3,4 \dagger}$, Shanzhou Huang ${ }^{1,2,3,5+}$, Qiang Zhao ${ }^{1,2,3}$, Changjun Huang ${ }^{1,2,3}$, Yunhua Tang ${ }^{1,2,3}$,

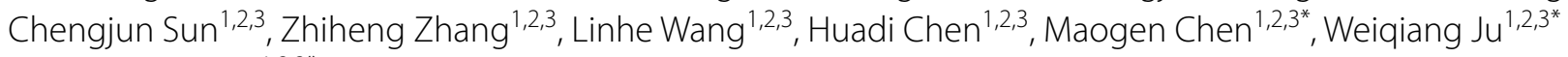
and Xiaoshun $\mathrm{He}^{1,2,3^{*}}$

\begin{abstract}
Background: MicroRNAs (miRNAs) play a pivotal role in hepatocellular carcinoma (HCC) progression and have been confirmed to participate in the carcinogenesis and development of HCC. However, the relationship between miR497-5p and HCC remains unclear.

Methods: Kaplan-Meier curve analysis and the log-rank test were used to investigate the efficacy of miR-497-5p on overall survival (OS) and disease-free survival (DFS) in patients with HCC. According to in vitro experiments, programmed cell death 4 (PDCD4) was a target of miR-497-5p by the dual-luciferase activity assay. The efficacy of PDCD4 on cell proliferation and metastasis in HCC was examined by transwell assays, CCK-8 assays and reverse transcription quantitative PCR (RT-qPCR). Additionally, we conducted a luciferase activity reporter assay to confirm the interaction between IncRNA XIST and miR-49-5p. Then, to evaluate the relationship between IncRNA XIST and miR-497-5p, several mechanistic experiments, including qRT-PCR, Western blotting, transwell assays and tumor xenograft assays, were performed.

Results: miR-497-5p was upregulated in HCC tissues, and high expression of miR-497-5p resulted in increases in tumor size and tumor number and a higher tumor-node-metastasis (TNM) stage and Edmondson grade in patients with HCC. Silencing miR-497-5p inhibited the proliferation and migration of HCC cells. PDCD4, which was downregulated in HCC tissues, was shown to be a target of miR-497-5p and was negatively correlated with the expression of miR-497-5p. IncRNA XIST was found to act as a miR-497-5p sponge and to regulate the level of PDCD4, which is targeted by miR-497-5p. IncRNA XIST was observed to be downregulated in the HCC tissues and positively correlated with the expression of PDCD4.
\end{abstract}

Conclusions: Our findings reveal that the XIST/miR-497-5p/PDCD4 axis participates in HCC development and that XIST could be used as a biomarker of HCC.

Keywords: IncRNA XIST, PDCD4, miR-497-5p, Proliferation, Migration, Hepatocellular carcinoma

\footnotetext{
*Correspondence: chenmg3@mail.sysu.edu.cn; weiqiangju@163.com; gdtrc@163.com

${ }^{\top}$ Yixi Zhang, Zebin Zhu and Shanzhou Huang contributed the same to

this article and are co-first authors

${ }^{1}$ Organ Transplant Center, The First Affiliated Hospital, Sun Yat-sen

University, No. 58 Zhongshan Er Road, Guangzhou 510080, China

Full list of author information is available at the end of the article
} 


\section{Background}

Hepatocellular carcinoma $(\mathrm{HCC})$ is one of the most deadly tumors in the world, especially in China [1] due to the high hepatitis B virus (HBV) infection rate [2-4]. Surgery and some other interventional therapies have greatly improved in recent years, but the outcomes of HCC patients remain poor [5]. Because of frequent recurrence and metastasis, HCC patients usually have poor prognosis [6]. Thus, exploring the mechanisms of $\mathrm{HCC}$ development is important for optimizing early diagnosis and treatment $[7,8]$.

Recent research has shown that the aberrant expression of noncoding RNAs (ncRNAs) is ubiquitous in different types of cancers, suggesting that ncRNAs play a key role in human carcinogenesis [9]. NcRNAs of less than 200 nucleotides are considered as small ncRNAs, i.e., microRNAs, while ncRNAs of more than 200 nucleotides are considered as long ncRNAs, i.e., lncRNAs [10]. Growing evidence suggests that the abnormal expression of lncRNAs is implicated in a variety of diseases, including cancer [11-13], and that some tumor-associated lncRNAs play key roles in the development and metastasis of HCC [14-16]. For example, lncRNA HULC [17], lncRNA EGFR [18], IncRNA HOST2 [19] and lncRNA Tim3 [20] accelerate $\mathrm{HCC}$ tumorigenesis and metastasis. However, lncRNA FTX [21] has been reported to inhibit HCC development and proliferation. lncRNA beta-Catm [16] is essential for the self-renewal of hepatocellular carcinoma stem cells and the proliferation of HCC tumors. MicroRNAs (miRNAs) constitute a group of small RNAs containing 18-25 nt. There is growing evidence that miRNAs are involved in various types of biological processes such as self-renewal, survival and tumor progression [22, 23]. In various studies, several miRNAs, such as miR-451, miR-128, miR-34 and miR-203, have been suggested to regulate cancer stemness and drug resistance in different types of cancer [24]. By targeting the $3^{\prime}$-untranslated regions (UTRs) of mRNAs, the expression of the target gene can be regulated posttranscriptionally, thereby affecting the regulation of cell proliferation, differentiation and apoptosis [1].

In the current study, we hypothesized that lncRNA X inactive-specific transcript (XIST) targets specific miRNAs and proteins to regulate HCC proliferation and migration, resulting in a poor prognosis in $\mathrm{HCC}$ patients. The biological roles of miR-497-5p in HCC development were explored, and we found that miR-497-5p was increased in both HCC tissues and cells, whereas lncRNA XIST was significantly decreased. We also observed that silencing of miR-497-5p could inhibit HCC progression in vitro. In addition, using bioinformatics methods, programmed cell death 4 (PDCD4) was predicted to be the target of miR-497-5p. Thus, we propose that IncRNA XIST inhibits HCC progression by targeting miR-497-5p and PDCD4 in vitro.

\section{Materials and methods Patient specimens}

In total, 77 patients with pathological diagnosis of $\mathrm{HCC}$ and who underwent hepatectomy at the 1st Affiliated Hospital of Sun Yat-sen University (SYSU) between January 2004 and December 2008 were included in this study. All samples were immediately frozen in a liquid nitrogen tank. The inclusion criteria were as follows: (1) radical resection; (2) no chemotherapy before surgery; (3) no distant metastasis; (4) survival for over 1 month after hepatectomy surgery; and (5) complete clinicopathological and follow-up data are available. In our study, the tumornode-metastasis (TNM) staging was evaluated based on the American Cancer Joint Commission (AJCC) Cancer Staging Manual, 7th Edition. The basic clinical information of the 77 HCC patients is shown in Table 1. All procedures carried out in studies involving human participants met the ethical standards of the Ethics Committee of the 1st Affiliated Hospital of Sun Yat-sen University and the 1964 Declaration of Helsinki and its subsequent revisions and amendments.

\section{Cell culture}

Human hepatic carcinoma cell lines (HepG2, HepB3, Huh7, SMMC-7721, MHCC-97L and Bel-7402), an immortalized hepatocyte cell line (LO2) and HEK293T cells were used in this study. All cells were purchased from the Institute of Cell Biology, Chinese Academy of Sciences (Shanghai, China). RPMI 1640 supplemented with 10\% fetal bovine serum (FBS) (HyClone, Shanghai, China), $100 \mathrm{U} / \mathrm{mL}$ penicillin and $100 \mu \mathrm{g} / \mathrm{mL}$ streptomycin (Gibco) or Dulbecco's modified Eagle's medium (DMEM, Sigma) was used as the cell culture medium, and all cells were cultured in a humidified chamber containing $5 \% \mathrm{CO}_{2}$ at $37^{\circ} \mathrm{C}$.

\section{Lentiviral vector transfection}

The human XIST full complementary DNA (cDNA) was amplified from HCC cells. The shRNA-luciferase (shluc) sequence was designed as a negative control. The target product was subcloned into pcDNA 3.1 (Invitrogen, Carlsbad, CA) using a lentivirus packaging vector and pMD2.G. In the medium containing $800 \mu \mathrm{g} / \mathrm{mL}$ G418 (Sigma-Aldrich), cells stably expressing XIST were cultured. Lipofectamine 2000 (Invitrogen) was used to introduce miR-497-5p mimics, inhibitors or negative controls into cells.

\section{CCK8 assay}

The cells were inoculated in 96-well plates overnight and infected for $48 \mathrm{~h}$ with a miRNA-497-5p inhibitor, an empty lentivirus vector, lentivirus (LV)-XIST or a LV negative control (NC) using Dojindo Molecular Technologies 
Table 1 Correlation between miR-497-5p expression and clinicopathological features of patients with HCC

$\begin{array}{llll}\begin{array}{l}\text { Clinicopathological } \\ \text { variables }\end{array} & \mathrm{n} & \frac{\mathrm{miR}-497-5 p \text { expression }}{\operatorname{Low}(53) \quad \text { High (24) }} & \end{array}$

Gender

Male

Female

Age (years)

$<50$

$\geq 55$

AFP (ng/mL)

Low, $<200$

High, $\geq 200$

Cirrhosis

Yes

No

$\mathrm{HBsAg}$

Negative

Positive

Tumor size $(\mathrm{cm})$

$<5$

$$
\geq 5
$$

Tumor number

Solitary

Multiple $(\geq 2)$

PVTT

No

Yes

TNM stage

$$
\text { I/II }
$$

III/IV

Edmondson grade

I/II

$>$ II

$\begin{array}{rr}66 & 47 \\ 11 & 6 \\ 33 & 20\end{array}$

$44 \quad 33$

$39 \quad 24$

$39 \quad 24$

$38 \quad 29$

3420

$43 \quad 33$

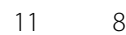

$66 \quad 45$

$28 \quad 16$

$49 \quad 37$

$54 \quad 34$

$23 \quad 19$

$63 \quad 43$

$14 \quad 10$

$47 \quad 27$

$30 \quad 26$

$30 \quad 23$

$47 \quad 30$

AFP alpha-fetoprotein determination, PVTT portal vein tumor thrombus, $H B s A g$ hepatitis $B$ surface antigen, TNM tumor node metastasis

(Tokyo, Japan) on days $0,1,2,3$ and 4 with a $100-\mu \mathrm{L}$ cell counting Kit-8 (CCK8). After incubating the cells with the CCK 8 reagent for $4 \mathrm{~h}$, the absorbance was measured at $450 \mathrm{~nm}$ by enzyme labeling (Bio-Tek, Winooski, VT).

\section{Transwell invasion assay}

A $200-\mu \mathrm{L}$ cell suspension was loaded into the upper chamber of 24 transwell permeability support chambers with 8-micron pores coated with $1 \mathrm{mg} / \mathrm{mL}$ Matrigel (Corning Incorporated, NY). The basement is equipped with $600 \mu \mathrm{L}$ of RPMI-1640 containing 10\% FBS. After that, the cells on the filter surface were fixed with $4 \%$ formaldehyde for $15 \mathrm{~min}$, stained with $0.5 \%$ crystal violet for $30 \mathrm{~min}$, and then observed using a microscope.

\section{Scratch wound assay}

The cells were inoculated in a 6-well plate, scraped through each hole with the tip of a sterile $10-\mu \mathrm{L}$ pipette and washed with phosphate buffered saline to remove any debris. After $24 \mathrm{~h}$, the cells that migrated to the empty space were observed.

\section{qRT-PCR}

RNAiso Plus (TaKaRa Biotechnology, Dalian, China) was used to extract total RNA. Prime Script ${ }^{\mathrm{T}}$ RT Master Mix was used to perform RNA reverse transcription. SYBR Premix Ex Taq II (TaKaRa Biotechnology) was used for qPCR. The primers used were as follows: for XIST (sense, 5'-AGCTCCTCGGACAGCTGTAA-3'; antisense, 5'-CTCCAGATAGCTGGCAACC-3); for PDCD4 (sense, 5'-TCG TCGTTACGATTGGTTAGTC-3'; antisense, 5'-GAAAAATCTCTA ACCCTTCTCGC-3); for miR497-5p: (sense, 5'-CCTTCAGCAGCACACTGTGG-3'; antisense, 5'-CAGTGCAGGGTCCGAGGTAT -3); for U6: (sense, 5'-CTCGCTTCGGCAGCACA-3'; antisense, 5'-TGGTGTCGTGGAGTCG-3'). An Applied Biosystems 7500 Real-Time PCR system (Applied Biosystems, Foster City, CA) was also utilized. The $2^{-\Delta \Delta \mathrm{Ct}}$ method was employed to analyze the relative gene expression levels.

\section{Western blot analysis}

Total protein was isolated from the cell lines and then resolved by $10 \%$ SDS-PAGE. Isolated proteins were transferred using a polyvinylidene fluoride (PVDF) membrane (Millipore, Billerica, MA). The membrane was incubated with a primary antibody, followed by incubation with secondary antibodies. The main antibodies included anti-PDCD4 (1:2000; Abcam of Cambridge University, Britain) and anti-glyceraldehyde 3-phosphate dehydrogenase (GAPDH) (1:1000, Abcam).

\section{Flow cytometry}

Cells were digested, washed with cold PBS, fixed with $70 \%$ cold ethanol and stored at $-20{ }^{\circ} \mathrm{C}$ for at least $48 \mathrm{~h}$. Before flow cytometry determination, fixed cells were washed and resuspended in $1 \mathrm{~mL}$ of PBS containing $10 \mathrm{mg} / \mathrm{mL}$ RNase A and were then incubated for $1 \mathrm{~h}$ at $37{ }^{\circ} \mathrm{C}$. Cell suspensions were stained with propidium iodide solution $(100 \mu \mathrm{g} / \mathrm{mL})$ in the dark for $30 \mathrm{~min}$. For each sample, 10,000 events were acquired, and cell cycle determinations were made by a FACS flow cytometer.

\section{Luciferase reporter gene assay}

For the luciferase reporter gene assay, $5 \times 10^{5}$ HEK293T cells were inoculated in a 24-well plate overnight. pmirGLO-PDCD4-WT or pmirGLO-lncRNA XISTWT reporter plasmids (150 ng each) and their mutant 
vectors were cotransfected into cells with $50 \mathrm{nM}$ mimic of miRNA-497-5p using Lipofectamine 2000 reagent. After $36 \mathrm{~h}$ of cell culture, the firefly and Renilla luciferase activities were determined by a double Luciferase Reporter Analysis System (Promega) based on the manufacturer's instruction manual. The relative luciferase activity was calculated based on the firefly/Renilla fluorescence ratio.

\section{Immunohistochemistry}

The tissue was fixed by $4 \%$ formalin and embedded in paraffin. The endogenous peroxidase activity was blocked, and each slide was subjected to antigen retrieval after peeling and rehydration. The slides were incubated overnight with antibodies against Ki67 (1:500, \#ab15580, Abcam) and PDCD4 (1:500, \#ab80590, Abcam) at $4{ }^{\circ} \mathrm{C}$. Slides were then incubated with a second antibody coupled with horseradish peroxidase (HRP) at $37{ }^{\circ} \mathrm{C}$ for $1 \mathrm{~h}$. The positive immune response rate was determined according to the ratio of positive cells.

\section{Detection of the xenotransplantation of tumors}

The animal experiment procedure was approved by the Animal Ethics Committee of the First Affiliated Hospital of Sun Yat-sen University. One-month-old female $\mathrm{BALB} / \mathrm{c}$ thymus-free nude mice were purchased from Shanghai Pharmaceutical Research Institute (Shanghai, China). HepG2 cells transfected with LV-XIST or LV-NC were subcutaneously implanted into the lateral abdomen of each nude mouse. After 1 week, the volume of the tumor was estimated using a caliper once a week for 5 weeks. The volume was calculated using the following formula: $\left(\mathrm{mm}^{3}\right)=0.5 \times$ length $\times$ width $^{2}$. All mice were euthanized, and the weights of the tumors were measured after 5 weeks. The levels of PDCD4 in the resected tumors were analyzed by Western blot and immunohistochemistry analyses. For immunohistochemical staining, two slices were stained with terminal TUNEL (Yeasen, Shanghai, China) according to the manufacturer's protocol. The cell nuclei were counterstained with 4,6-diamidino-2-phenylindole (DAPI, Sigma). TUNEL-stained slides were visualized under a fluorescence microscope (Zeiss Axio Observer. Z1).

\section{Statistical analysis}

Student's t-test was used to analyze the differences between the two experimental groups. One-way ANOVA was used to analyze the differences among more than two different groups. Pearson's correlation coefficient was used to evaluate the correlations between different groups. $X^{2}$ test or Fisher's exact test were used to determine whether the target was correlated with the clinical pathological parameters. SPSS 24.0 software (Chicago, IL, USA) was used for the statistical analysis. A $p<0.05$ was considered statistically significant.

\section{Results \\ Correlation between miRNA-497-5p expression and clinicopathological features in HCC patients}

To illustrate the role of miR-497-5P in the invasive progression of HCC, the expression of miR-497-5p and the basic information, such as clinical pathological features of patients with HCC, are displayed in Table 1 . The median age of the patients was 57.5 years (31-75 years), and the median size of tumors was $6 \mathrm{~cm}(2-18 \mathrm{~cm})$. The results showed that high expression of miRNA-497-5p was correlated with the Edmondson stage $(p=0.026)$, tumor size $(p=0.019)$ and TNM stage $(p=0.011)$. However, there was no significant correlation between miRNA-497-5p and number of tumors $(p=0.111)$, fetoprotein (AFP) $(p=0.162)$, gender $(p=0.269)$, age $(p=0.177)$, alpha cirrhosis $(p=0.092)$, HBV infection $(p=0.761)$ or portal vein cancer thrombus (PVTT) $(p=0.545)$.

\section{High levels of miR-497-5p predict a poor prognosis}

We further studied the relationship between the level of miRNA-497-5p and survival time in 77 HCC patients to clarify the prognostic value of miRNA-497-5p. The level of miRNA-497-5p was detected by qRT-PCR, and the results showed that compared with the adjacent normal tissues, miRNA-497-5p was significantly upregulated in the HCC tissues (Fig. 1a). Significant differences were found in the overall survival (OS) and disease-free survival (DFS) between different miRNA-497-5p expression groups (the high and low group) $(p<0.001$ and $p<0.001)$, as shown in Fig. 1b, c. In addition, univariate and multivariate Cox proportional risk regression analyses were used to determine the independent prognostic factors of OS and DFS in HCC patients. The results showed that the Edmondson grade (hazard ratio [HR]: 2.768; 95\% confidence interval [CI] 1.206-6.352 for III + IV vs. I + II, $p=0.016$ ) and lower miRNA-497-5p expression (HR 0.016 ; $95 \%$ CI $0.242-0.767, p=0.015$ ) were significant independent prognostic factors of OS (Table 2). In addition, the results demonstrated that the Edmondson grade (HR, 5.442; 95\% CI 2.778-9.714, $\mathrm{p}=0.001$ ) and lower miRNA-497-5p expression (HR, 0.090; 95\% CI 0.018$0.461, p=0.004)$ were significant prognostic factors of DFS in patients with HCC (Table 3).

\section{miR-497-5p was upregulated in HCC and could promote cell proliferation and migration in HCC}

Given the increased expression of miR-497-5p in the $\mathrm{HCC}$ tissues, we explored the expression levels of miR497-5p in HCC cell lines (Huh7, Bel-7402, HepG2, 

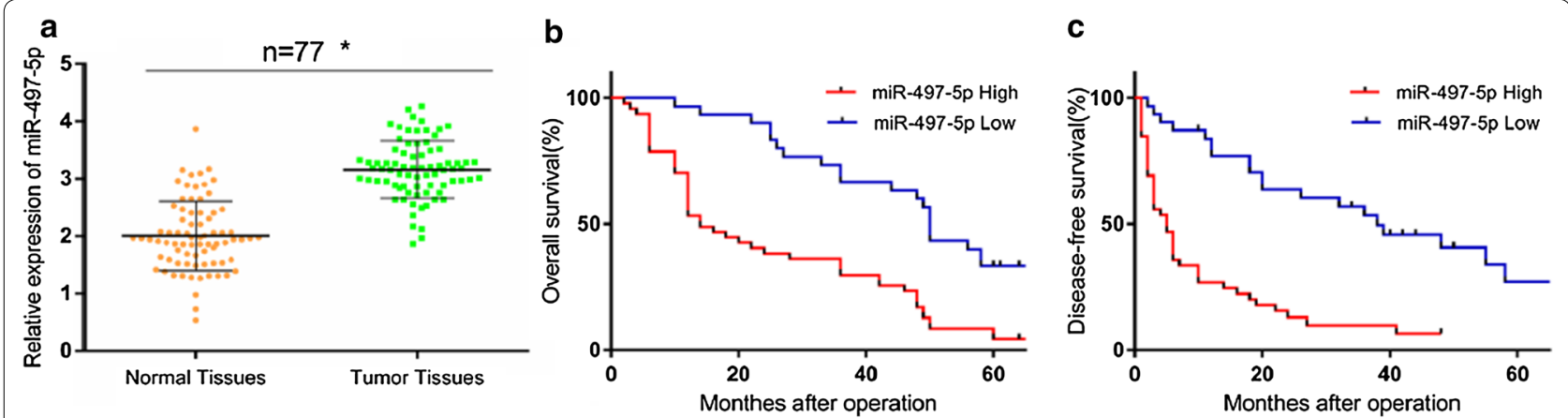

Fig. 1 miR-497-5p was increased in HCC and correlated with prognosis in HCC patients. a Expression levels of miR-497-5p in HCC tissues and adjacent normal tissues were measured by qRT-PCR. $\mathbf{b}$ The overall survival of HCC patients with high or low expression of miR-497-5p were evaluated by Kaplan-Meier analysis. c The disease-free survival of HCC patients with high or low expression of miR-497-5p were evaluated by Kaplan-Meier analysis. Error bars represent the mean \pm SD from three independent experiments. ${ }^{*} p<0.05$. miR microRNA-497-5p

Table 2 Univariate and multivariate Cox regression analyses of risk factors associated with overall survival

\begin{tabular}{|c|c|c|c|c|c|c|}
\hline \multirow[t]{2}{*}{ Variables } & \multicolumn{3}{|c|}{ Univariate analysis } & \multicolumn{3}{|c|}{ Multivariate analysis } \\
\hline & HR & $95 \% \mathrm{Cl}$ & p-value & HR & $95 \% \mathrm{Cl}$ & p-value \\
\hline miR-497-5p expression (low vs. high) & 4.603 & $1.914-9.465$ & 0.001 & 4.116 & $1.542-8.767$ & 0.005 \\
\hline Gender (male vs. female) & 0.656 & $0.325-1.326$ & 0.241 & & & \\
\hline Age $(<50$ vs. $\geq 55)$ & 0.609 & $0.370-1.005$ & 0.052 & & & \\
\hline HBsAg (negative vs. positive) & 0.918 & $0.481-1.753$ & 0.796 & & & \\
\hline Cirrhosis (yes vs. no) & 1.435 & $0.886-2.325$ & 0.142 & & & \\
\hline $\operatorname{AFP}(<200 \mathrm{ng} / \mathrm{mL}$ vs. $\geq 200 \mathrm{ng} / \mathrm{mL})$ & 1.766 & $1.092-2.857$ & 0.020 & 1.548 & $0.924-2.592$ & 0.097 \\
\hline Tumor size $(<5 \mathrm{~cm}$ vs. $\geq 5 \mathrm{~cm})$ & 1.651 & $1.007-2.707$ & 0.047 & 0.718 & $0.387-1.331$ & 0.293 \\
\hline Tumor number (single vs. multiple) & 2.184 & $1.296-3.680$ & 0.003 & 1.105 & $0.585-2.088$ & 0.758 \\
\hline PVTT (no vs. yes) & 3.487 & $1.852-6.564$ & 0.001 & 1.229 & $0.537-2.816$ & 0.626 \\
\hline TNM stage (I/II vs. III/IV) & 3.331 & $1.989-5.578$ & 0.001 & 2.401 & $0.791-2.285$ & 0.171 \\
\hline Edmondson grade (I/II vs. > II) & 4.384 & $2.561-7.505$ & 0.001 & 2.768 & $1.206-6.352$ & 0.016 \\
\hline
\end{tabular}

AFP alpha-fetoprotein determination, PVTT portal vein tumor thrombus, HBsAg hepatitis B virus, TNM tumor node metastasis, $H R$ hazard ratio, $C l$ confidence interval

Hep3B, MHCC-97L and SMMC-7721) and the hepatocyte cell line L02 (HL-7702) and discovered that miR497-5p was upregulated in the HCC cell lines (shown in Fig. 2a). Then, we transfected Huh7 and HepG2 cells with a miR-497-5p inhibitor to assess the biological effect of miR-497-5p in HCC cells. Wound healing and CCK8 assays revealed that the miR-497-5p-inhibitor suppressed the proliferative capacity of the HepG2 and Huh7 cells (Fig. 2b, c). In addition, compared with the control group, the migration of the Huh7 and HepG2 cells transfected with miRNA-497-5p-inhibitors was also decreased (Fig. 2d). To explore the roles in cellular activity, the expression levels of several cell cycle regulatory markers were further examined by Western blot analysis. The results showed that Cyclin D1, CDK4 and Cyclin E expression levels were inhibited while that of P16 was elevated in the miR-497-5p inhibitor group. In comparison, miR-NC significantly increased the expression levels of Cyclin D1, CDK4 and Cyclin E and decreased the expression of P16 (Fig. 2e).

\section{PDCD4 is a direct target of miR-497-5p}

To identify the underlying mechanism of miR-497-5p in $\mathrm{HCC}$, the miRanda database was searched to make a prediction of the potential target for miR-497-5p. PDCD4 was suggested as a potential target of miRNA497-5p due to the presumptive binding sequence of microRNA-497-5p in its $3^{\prime}$-UTR (shown in Fig. 3a). To confirm this prediction, double luciferase assay assays were performed. The results are shown in Fig. 3b; miR497-5p inhibited the relative luciferase activity in cells containing the wild-type (WT) 3'-UTR of the PDCD4 construct but did not alter that in cells containing the mutated (Mut) 3'-UTR of the PDCD4 construct. To further verify that PDCD4 is a target of miRNA-497-5p, we surveyed the expression of PDCD4 in miR-497-5p- or 
Table 3 Univariate and multivariate Cox regression analyses of risk factors associated with disease-free survival

\begin{tabular}{|c|c|c|c|c|c|c|}
\hline \multirow[t]{2}{*}{ Variables } & \multicolumn{3}{|c|}{ Univariate analysis } & \multicolumn{3}{|c|}{ Multivariate analysis } \\
\hline & HR & $95 \% \mathrm{Cl}$ & p-value & HR & $95 \% \mathrm{Cl}$ & p-value \\
\hline miR-497-5p expression (high vs. low) & 3.358 & $1.516-7.408$ & 0.001 & 3.197 & $1.264-6.121$ & 0.004 \\
\hline Gender (male vs. female) & 0.379 & $0.116-1.237$ & 0.108 & & & \\
\hline Age $(<50$ vs. $\geq 55)$ & 0.501 & $0.246-1.024$ & 0.058 & & & \\
\hline HBsAg (negative vs. positive) & 1.104 & $0.429-2.838$ & 0.838 & & & \\
\hline Cirrhosis (no vs. yes) & 2.181 & $1.092-4.355$ & 0.027 & 1.065 & $0.455-2.497$ & 0.884 \\
\hline $\operatorname{AFP}(<200 \mathrm{ng} / \mathrm{mL}$ vs. $\geq 200 \mathrm{ng} / \mathrm{mL})$ & 2.311 & $1.182-4.518$ & 0.014 & 0.951 & $0.371-2.135$ & 0.616 \\
\hline Tumor size $(<5 \mathrm{~cm}$ vs. $\geq 5 \mathrm{~cm})$ & 3.523 & $1.595-7.782$ & 0.002 & 1.584 & $0.591-4.245$ & 0.360 \\
\hline Tumor number (single vs. multiple) & 1.652 & $0.818-3.337$ & 0.162 & & & \\
\hline PVTT (no vs. yes) & 3.971 & $1.798-8.768$ & 0.001 & 1.651 & $0.759-3.590$ & 0.206 \\
\hline TNM stage (I/II vs. III/IV) & 2.945 & $1.511-5.739$ & 0.002 & 1.570 & $0.609-4.046$ & 0.351 \\
\hline Edmondson grade (I/II vs. >II) & 3.384 & $1.419-9.660$ & $<0.01$ & 5.442 & $2.778-9.714$ & 0.001 \\
\hline
\end{tabular}

AFP alpha-fetoprotein determination, PVTT portal vein tumor thrombus, HBsAg hepatitis B surface antigen, TNM tumor node metastasis, HR hazard ratio, CI confidence interval

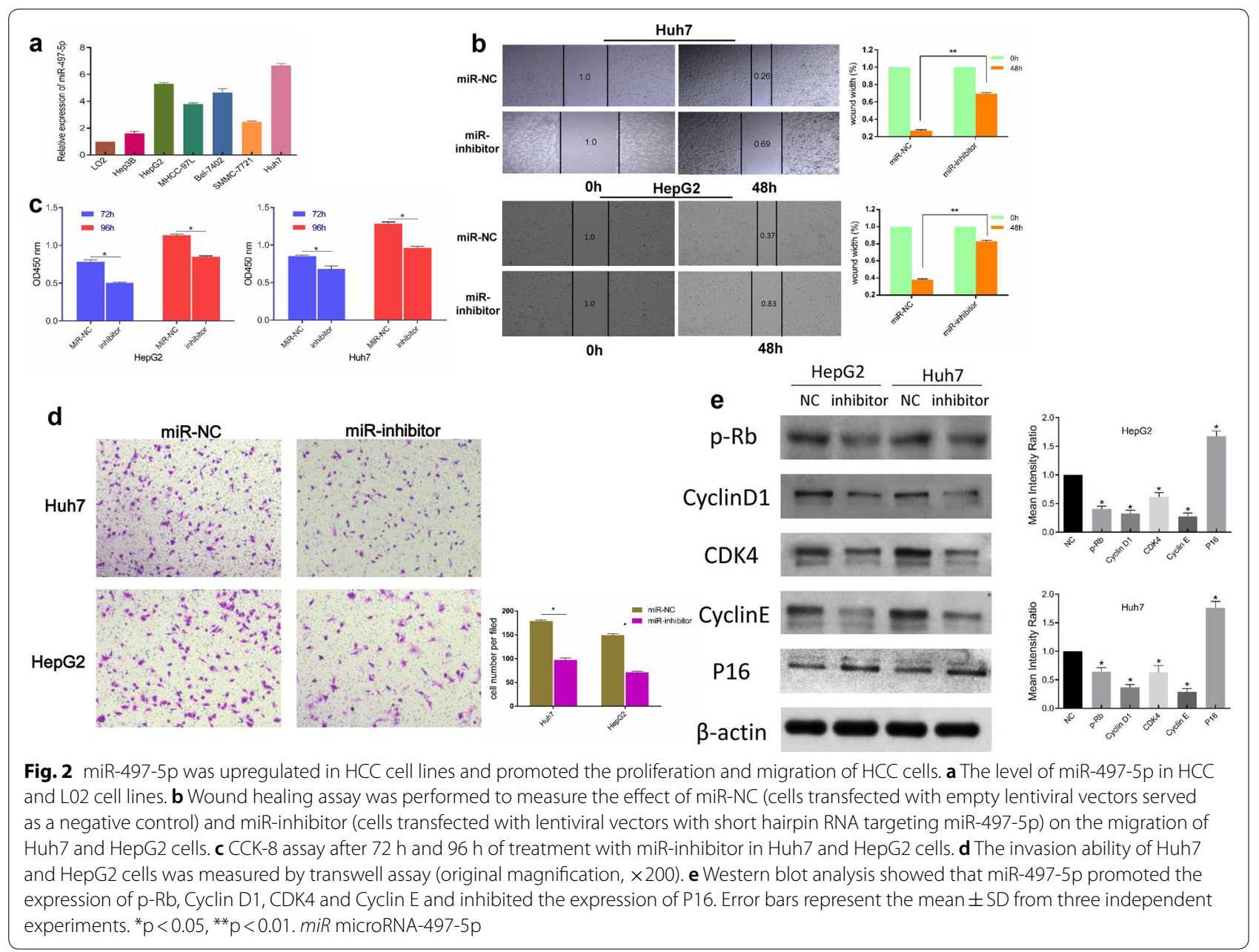


NC miRNA-transfected cells. As expected, the protein expression of PDCD4 was increased by the miR-497-5pinhibitor (Fig. 3c, d), revealing that PDCD4 is a direct target of miR-497-5p in HCC.

\section{PDCD4 downregulates and inhibits the proliferation and migration of HCC cells}

Since PDCD4 was found to be a direct target of miR497-5p, the expression levels of PDCD4 in 77 pairs of HCC samples were measured by qRT-PCR. Compared with the adjacent normal tissues, PDCD4 was downregulated in 57 (74.0\%) HCC tissues ( $<<0.001$, Fig. 3e). Western blot analysis further confirmed the significant downregulation of PDCD4 in HCC (Fig. 3f). Additionally, we examined the expression levels of PDCD4 and miR-497-5p and found that they were negatively correlated (Fig. 3g). Subsequently, we overexpressed PDCD4 in HepG2 and Huh7 cells (Fig. 4a) and discovered that overexpression of PDCD4 suppressed cell proliferation and migration (Fig. 4b, c). Furthermore, knocking down PDCD4 enhanced the cell proliferation and migration of the HepG2 and Huh7 cells (Fig. 4d, f). To investigate the effects of PDCD4 on apoptosis and the cell cycle in HCC cells, we detected the expression levels of Cyclin D1, CDK4, Cyclin E, P16 and apoptosis markers such as cleaved caspase 3 , caspase 8 , caspase 9 , poly ADP ribose polymerase (PARP) in HepG2 and Huh7 cells with upregulation or downregulation of PDCD4. Flow cytometry was then utilized for cell cycle analysis. The results of this experiment showed that si-PDCD4 significantly increased the expression levels of Cyclin D1, CDK4 and Cyclin E and decreased the expression of P16 (Fig. 4g). As shown in Fig. 4h, cells were blocked in G2 phase after interfering with PDCD4. The si-PDCD4 group also exhibited a decreased apoptotic rate and reduced protein expression levels of cleaved caspase 3, 8 and 9 and PARP (Fig. 4i). All of these results indicate that miR-497-5p can promote cell proliferation and migration in $\mathrm{HCC}$ by targeting PDCD4.

\section{XIST and miR-497-5p are negatively related in HCC}

To investigate the underlying biological mechanism by which XIST has an effect on HCC development, we carried out a bioinformatics analysis. We used miRanda (http://www.microrna.org) and StarBase v3.0 (http:// starbase.sysu.edu.cn/) to predict possible lncRNAs interacting with miR-497-5p. The lncRNA XIST, which contained complementary sequences to the seed region of miR-497-5p, was predicted. The predicted binding sites of XIST and miR-497-5p are shown in Fig. 5a. To validate the interaction between miR-497-5p and XIST, we carried out luciferase reporter assays. The plasmids of pmirGLO-lncRNA XIST-WT and pmirGLO-lncRNA XIST-MUT were cotransfected into HepG2 and Huh7 cells with miRNA-497-5p or miRNA-control. The results indicated that ectopic expression of microRNA-497-5p

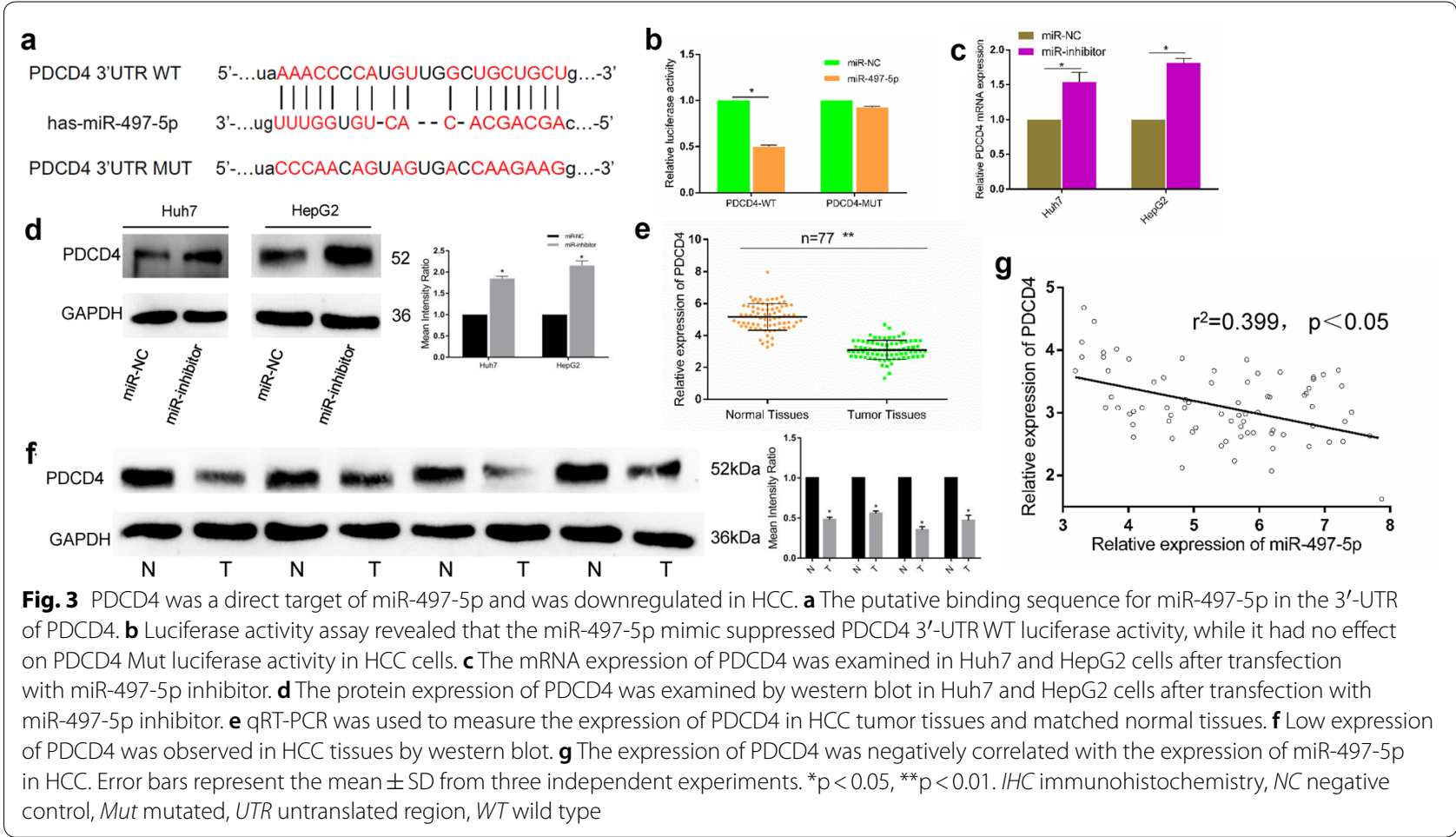




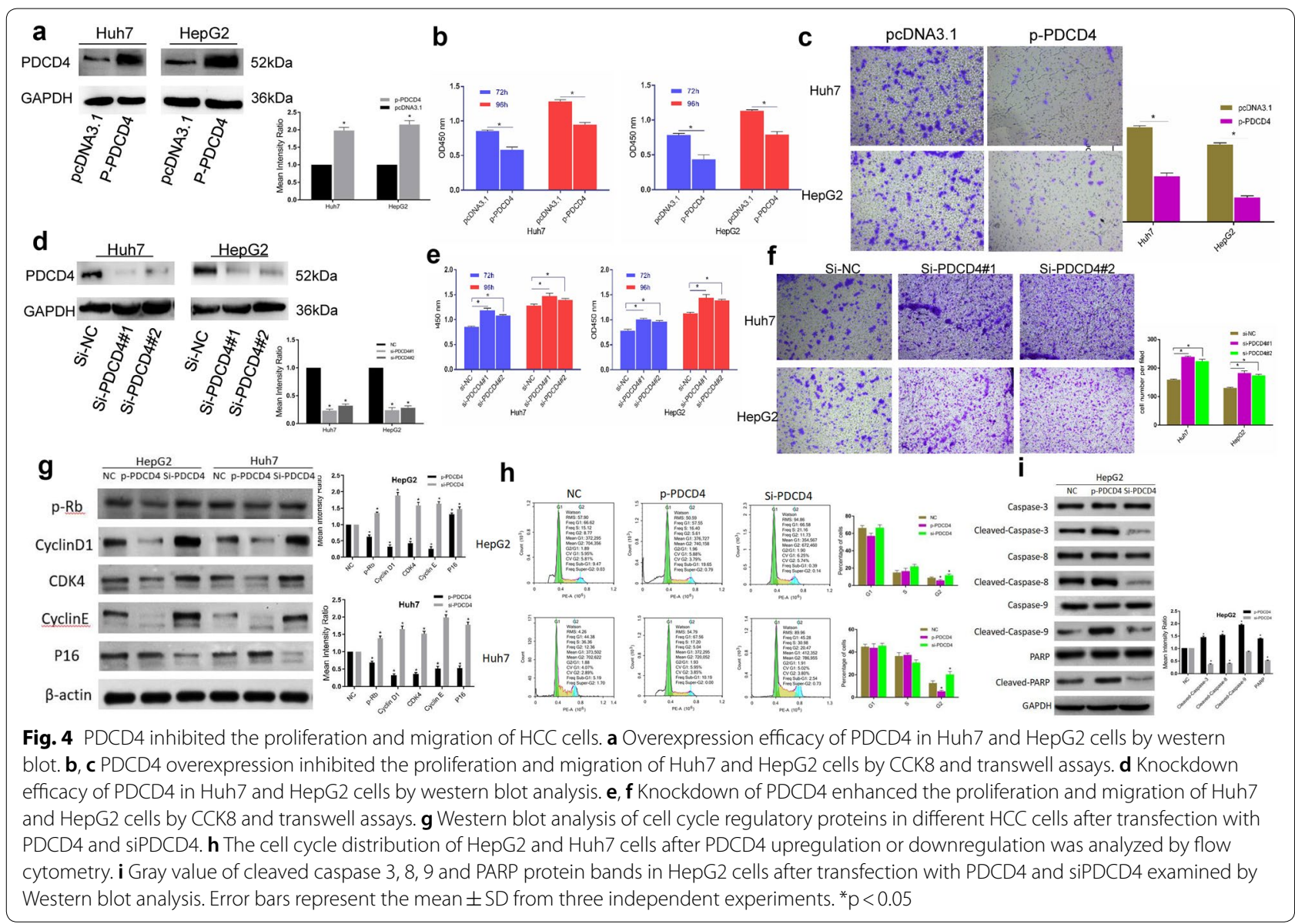

resulted in a significant downregulation in the luciferase activity of XIST-WT, but not XIST-MUT, in Huh7 and HepG2 cells (Fig. 5b). The qRT-PCR results showed that IncRNA XIST was significantly downregulated in the 77 HCC tissues compared with the matched adjacent normal tissues (Fig. 5c). In addition, a significant negative correlation between the expression of miR-497-5p and XIST was found in HCC tissues (Fig. 5d). To further pinpoint the regulatory relationship between miR-497-5p and XIST, we transfected HepG2 and Huh7 cells with XIST-mimic, XIST-MUT, XIST-WT or respective controls. XIST was obviously increased after transfection with the XISTmimic (Fig. 5e). Moreover, the level of miR-497-5p was considerably elevated in HepG2 and Huh7 cells following the introduction of XIST-WT, but there was no distinct change in the cells treated with XIST-MUT (Fig. 5f). These data indicate that a direct interaction exists between XIST and miR-497-5p in HCC.

\section{LncRNA XIST regulates the proliferation and migration of HCC cell lines through the miRNA-497-5p axis}

After confirming that lncRNA XIST could bind miR497-5p and is positively correlated with PDCD4 expression, we hypothesized that lncRNA XIST plays an equally vital role in HCC. We then overexpressed lncRNA XIST and found that the proliferation and migration of the HepG2 and Huh7 cells were obviously suppressed by LV-XIST, but there was no difference in the cells transfected with the XIST negative control (NC) (Fig. 6a, b).

If a lncRNA serves as a molecular sponge of miRNA, then its upregulation could lead to an elevation in the miRNA targets. To determine whether lncRNA XIST regulated the proliferation and migration in HepG2 and Huh7 cells through targeting PDCD4 by sponging miR497-5p, we overexpressed lncRNA XIST and discovered that both the PDCD4 gene and protein expression levels were increased (Fig. 6c, d). We conducted rescue experiments to determine whether lncRNA XIST regulates cell proliferation and PDCD4 expression via miR-497-5p in HCC. The reductions in the proliferation and migration of the Huh7 and HepG2 cells generated by lncRNA XIST overexpression could be rescued by the miR-497-5p mimic (Fig. 6e, f). The inhibition of PDCD4 by lncRNA XIST could also be rescued by overexpressing miR497-5p (Fig. 6g). Altogether, the above data indicate that 

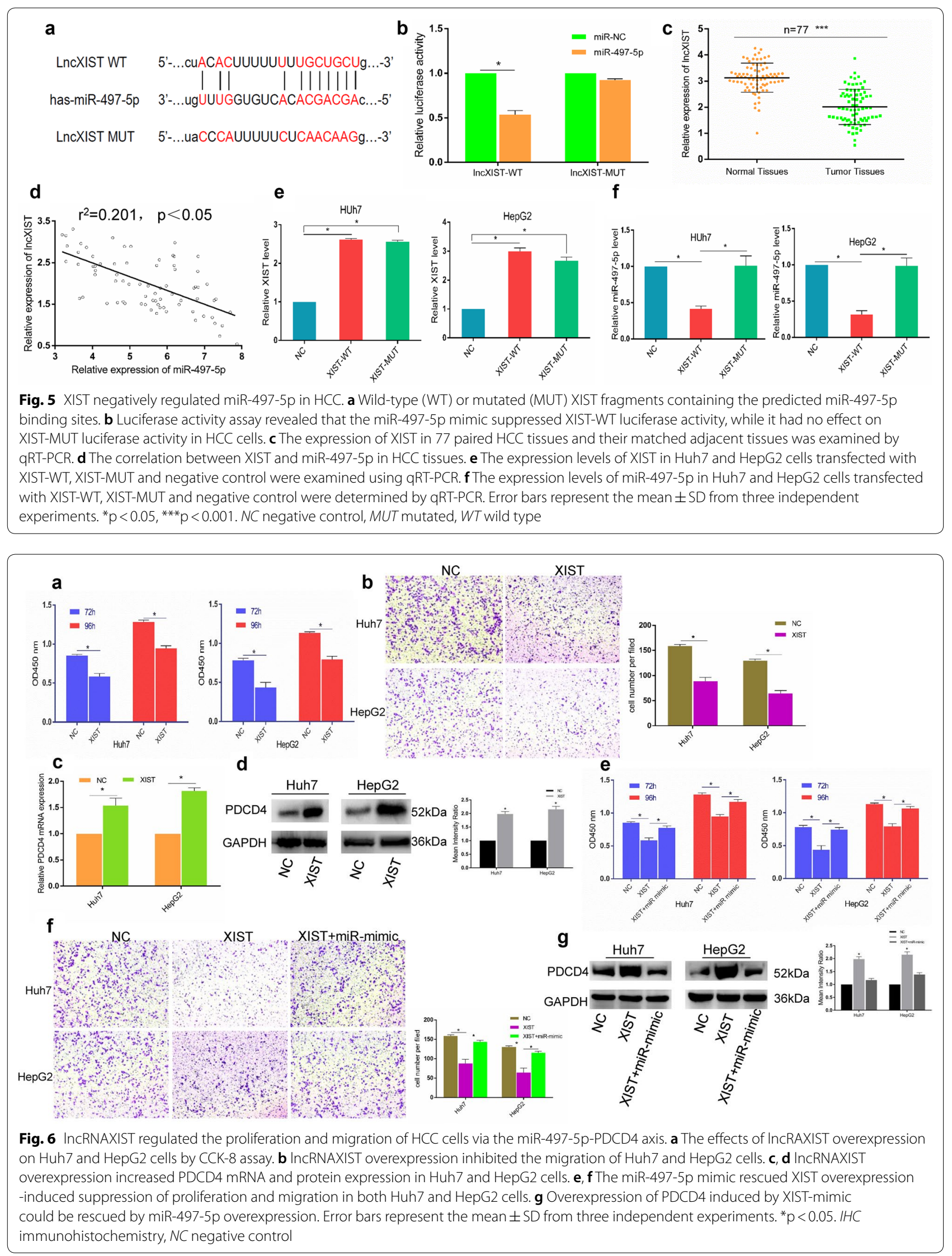
lncRNA XIST regulates the cell proliferation and migration in HCC through the miR-497-5p-PDCD4 axis.

\section{Overexpression of IncRNA XIST inhibited tumor growth in vivo}

We verified that lncRNA XIST overexpression inhibited the viability of Huh7 and HepG2 cells in vitro. Therefore, we propose that IncRNA XIST performs the same function in vivo. Twelve mice were randomly divided into two groups: six mice were injected with HepG2 cells with stable lncRNA XIST overexpression (LV-lncRNA XIST-HepG2), and six mice were injected with cells transfected with the control vector (LV-NC-HepG2). The tumor volume was measured weekly for consecutive weeks. After 5 weeks, the mice were sacrificed by anesthesia, and the xenograft tumors were collected for further analysis. Consistent with the in vitro results, the lncRNA XIST overexpression inhibited HepG2 xenograft growth in vivo (Fig. 7a, c). The proliferation marker Ki67 was reduced in the LV-lncRNA XIST-HepG2 xenografts compared with that in the LV-NC-HepG2 xenografts, indicating that lncRNA XIST inhibited tumor growth. Furthermore, Western blot and IHC analyses further confirmed the overexpression of PDCD4 in the LV-lncRNA XIST-HepG2 xenografts in vivo (Fig. 7d, e). Consistently, the terminal deoxynucleotidyl transferase (TdT)-mediated dUTP nick end labeling (TUNEL) staining results were in agreement with the above results: many more FITC-positive apoptotic cells were observed in the XIST-treated group (Fig. 7f). A schematic diagram is depicted in Fig. 8 and summarizes the key findings of our study. Overall, our findings indicate that lncRNA XIST can regulate $\mathrm{HCC}$ tumor growth through the miR497-5p-PDCD4 axis.

\section{Discussion}

The prognosis of HCC patients is typically poor. The main cause of the poor prognosis of these patients is the high possibility of metastasis and recurrence after operation. Previous studies have shown that advanced TNM staging is a vital independent predictor of poor prognosis in HCC. In this study, we also found that a late TNM stage and high expression of miRNA-497-5p were independent prognostic factors of OS and DFS in HCC through a Cox proportional hazard analysis. Additionally, the results of Kaplan-Meier curve analysis showed that patients with high levels of miRNA-497-5p had a worse prognosis than those with low miRNA-497-5p expression. In addition, a higher level of miR-497-5p was associated with malignant behavior in HCC (Table 1), including a larger tumor size, later TNM stage, and higher PVTT and Edmondson grade (Table 2). These findings suggest that the highly expressed miRNA-497-5p is involved in the progression of HCC.

Recent studies have shown that ncRNAs could serve as molecular markers of cancers, including $\operatorname{HCC}[25,26]$. Therefore, it is important to clarify the roles of miRNAs and IncRNAs in the prognosis of HCC. In this study, miRNA-497-5p was upregulated in HCC cells, while
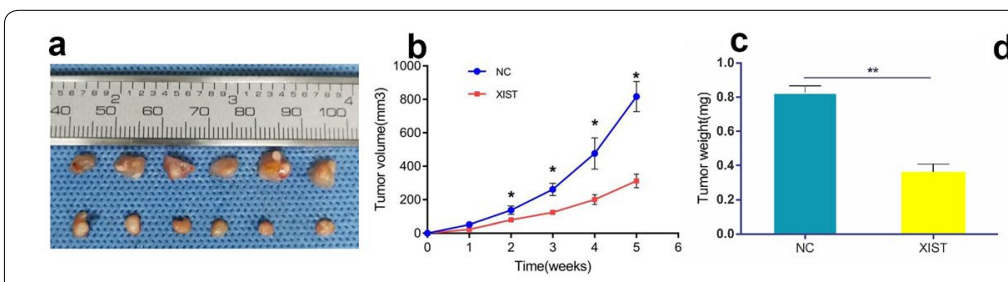

d

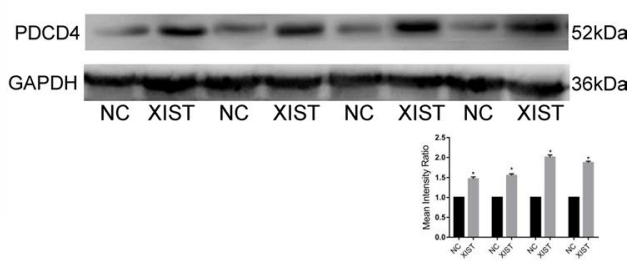

e NC PDCD4
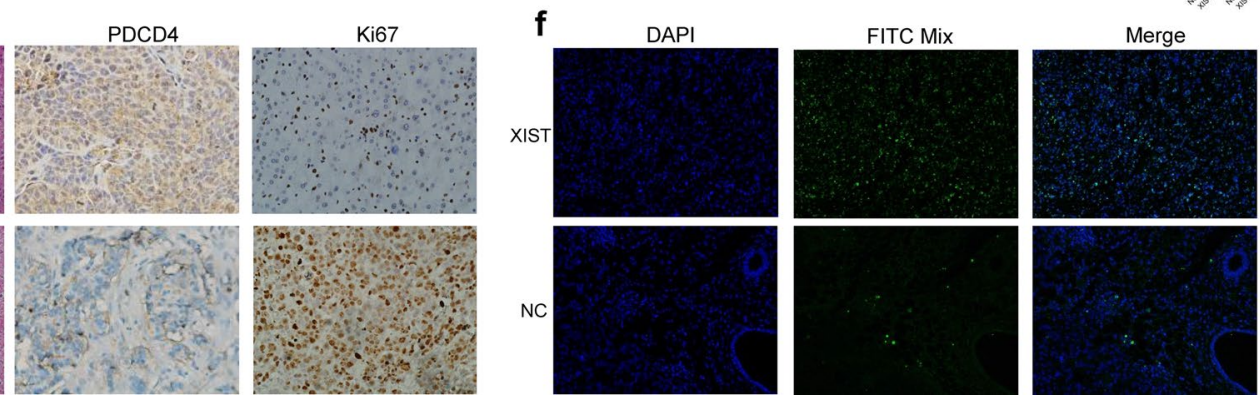

Fig. 7 Overexpression of IncRNAXIST inhibited tumor growth in vivo. a The xenograft tumors were obviously inhibited by IncRNAXIST. b, c Tumor volume and tumor weight of the xenografts were significantly suppressed by IncRNAXIST-mimic. d IncRNAXIST-mimic upregulated PDCD4 expression in vivo by western blot analysis. e The expression of PDCD4 and Ki67 in the xenograft tumors was examined by IHC. Error bars represent the mean \pm SD from three independent experiments. $\mathbf{f}$ Terminal deoxynucleotidyl transferased UTP nick end labeling (TUNEL) of specific tumor tissues at 21 days post-treatment. Green fluorescence indicated TUNEL-positive apoptotic cells; blue fluorescence indicated DAPI-stained nuclei. ${ }^{* *} \mathrm{p}<0.01$. IHC immunohistochemistry, NC negative control, Mut mutated, UTR untranslated region, WT wild type 

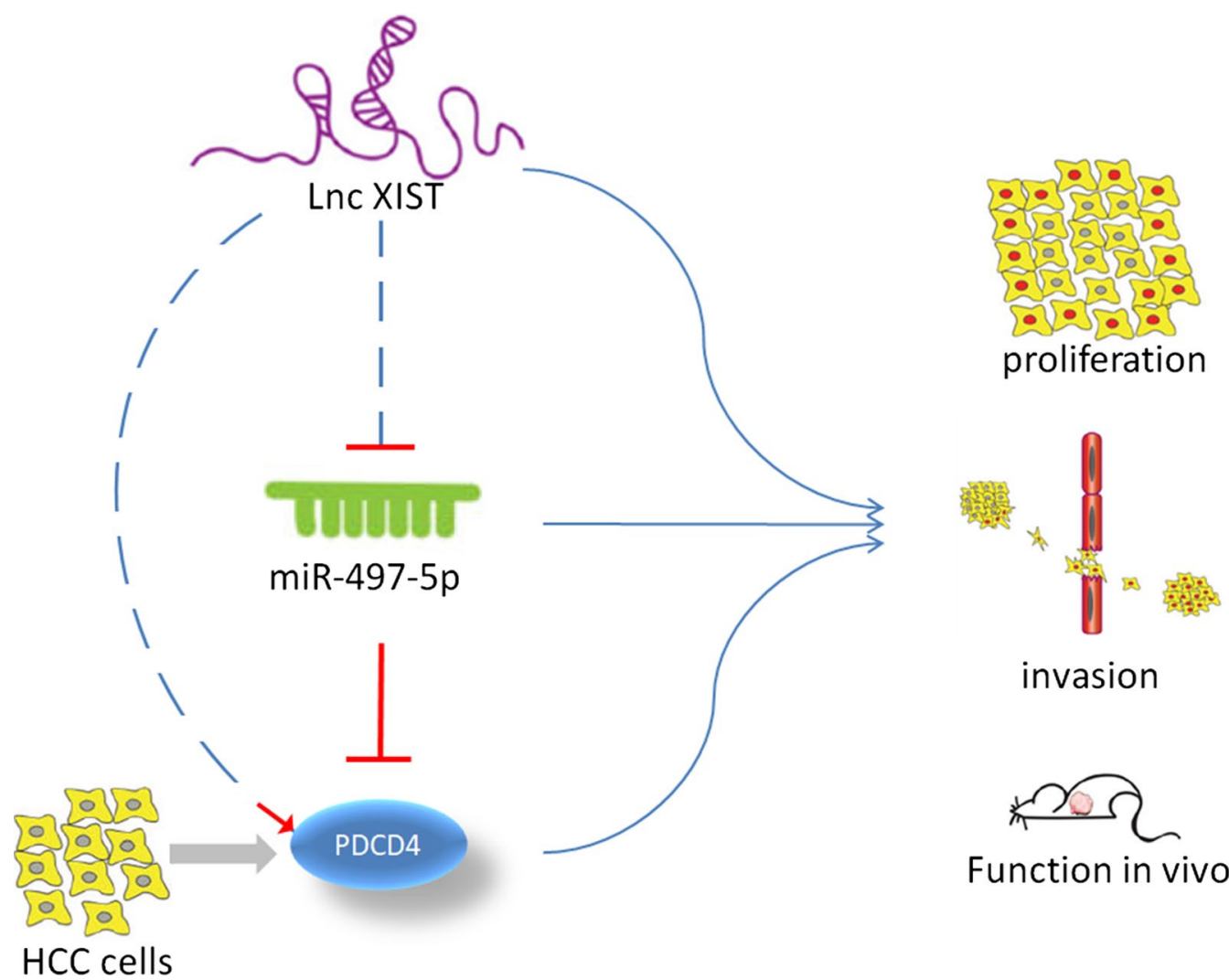

Function in vivo

Fig. 8 Schemtic diagram shows how XIST regulates HCC cells proliferation and invasion by miR-497-5p as a ceRNA

lncRNA XIST was downregulated. The overexpression of XIST could inhibit the development of HCC and rescue the miR-497-5p mimic-induced progression of HCC in vitro. In addition, using bioinformatics methods, we predicted that PDCD4 is a target of miRNA-497-5p. Therefore, we propose that the XIST/miR-497-5p/ PDCD4 axis participates in the development of HCC. We found that the level of lncRNA XIST was lower in HCC cells and that overexpression of lncRNA XIST inhibited the progression of HCC. The above results indicate that lncRNA XIST plays a major role in inhibiting HCC development.

Studies have demonstrated that XIST could modulate proliferation and apoptosis in osteoarthritis chondrocytes [27]. In addition, it has been reported that the level of IncRNA XIST in many cancer patients is reduced. For example, in ovarian cancer, the upregulation of lncRNA XIST has anticancer effects due to the inverse downregulation of has-miR-214-3p [28]. In breast cancer, XIST could inhibit proliferation and migration by activating MSN-c-Met and reprogramming microglia to promote brain metastasis [29]. Importantly, the elevation in miR497-5p may contribute to the XIST-mediated inhibition of liver cancer cell growth. In the current study, our findings provide novel evidence to support this action. This affirmation stems from several findings: (1) XIST is negatively associated with miR-497-5p levels in human HCC tissues and cells; (2) XIST suppressed miR-497-5p expression by targeting PDCD4; and (3) XIST increased the expression of PDCD4 by decreasing miR-497-5p in human liver cancer tissues.

PDCD4 is involved in regulating apoptosis, is located on human chromosome 10q25.2 and is considered a novel tumor suppressor gene [30]. While the PDCD4 protein is usually located in the nucleus, when the cell microenvironment changes, such as during malignant proliferation, PDCD4 can be transferred to the cytoplasm through nuclear export signals [31]. PDCD4 can also bind ribosomes directly, affect the posttranscriptional translation process, and lead to cell apoptosis in cancer [32]. In this study, we predicted that PDCD4 was a target gene of miRNA-497-5p by bioinformatics and that the expression level of PDCD4 could be inhibited by miR497-5p. In addition, we found that XIST could attenuate the level of PDCD4 and that PDCD4 expression could be inhibited by overexpressed miR-497-5P in HCC cells. 


\section{Conclusions}

Altogether, the results of this study indicate that XIST may have an inhibitory effect on HCC. In this work, we confirmed the potential mechanism of the XIST/ miR-497-5P/PDCD4 axis in HCC cells and determined that XIST overexpression could inhibit the progression of HCC. In addition, we found a negative correlation between XIST and miRNA-497-5p. We focused on PDCD4 because it is a target of miR-497-5p. Our findings reveal that the XIST/miR-497-5p/PDCD4 axis participates in the development of HCC and that XIST may be considered a potential biomarker of HCC.

\begin{abstract}
Abbreviations
HCC: hepatocellular carcinoma; IncRNAs: long noncoding RNAs; 3'-UTR : 3'-untranslated region; HBV: hepatitis B virus; IHC: immunohistochemistry; AFP: alpha-fetoprotein; TNM: tumor-node-metastasis; qRT-PCR: quantitative real-time PCR.
\end{abstract}

\section{Acknowledgements}

Not applicable.

\section{Authors' contributions}

$Y Z$ and $M C$ contributed to the design and writing of the study; ZeZ and ZhZ acquired the data; $Y T, S H, L W, H C$ and CS performed the experiments; MC and WJ contributed to the data analysis; and $\mathrm{YZ}$ and $\mathrm{XH}$ contributed to drafting or critically modifying important content and eventually approved the version to be published. All authors read and approved the final manuscript.

\section{Funding}

This study was supported by the National Natural Science Foundation of China (81401324 and 81770410), the Guangdong Provincial International Cooperation Base of Science and Technology (Organ Transplantation) (2015B050501002), the Guangdong Provincial Natural Science Funds for Distinguished Young Scholars (2015A030306025), the Special Support Program for Training High-Level Talent in Guangdong Province (2015TQ01R168), the Pearl River Nova Program of Guangzhou (201506010014), and the Scientific Program for Young Teachers of Sun Yat-sen University (16ykpy05), China.

\section{Availability of data and materials}

The data used and analyzed in this study are available from the corresponding author upon request.

\section{Ethics approval and consent to participate}

This study was approved by the Research Ethics Committee of the First Affiliated Hospital of SYSU. All procedures in studies involving human participants were performed in accordance with the ethical standards of the Ethics Committee of the First Affiliated Hospital of SYSU and the 1964 Declaration of Helsinki and its later amendments or revisions.

\section{Consent for publication}

The authors agree to publication.

\section{Competing interests}

The authors declare that they have no competing interests.

\footnotetext{
Author details

1 Organ Transplant Center, The First Affiliated Hospital, Sun Yat-sen University, No. 58 Zhongshan Er Road, Guangzhou 510080, China. ${ }^{2}$ Guangdong Provincial Key Laboratory of Organ Donation and Transplant Immunology, Guangzhou, China. ${ }^{3}$ Guangdong Provincial International Cooperation Base of Science and Technology, Guangzhou, China. ${ }^{4}$ Organ Transplant Center, The First Affiliated Hospital of USTC, Division of Life Sciences and Medicine, University of Science and Technology of China, Hefei 230001, Anhui, China. ${ }^{5}$ Department of General Surgery, Guangdong General Hospital, Guangdong Academy of Medical Sciences, Guangzhou, China.
}

Received: 16 February 2019 Accepted: 15 July 2019

Published online: 29 July 2019

\section{References}

1. Rupaimoole R, Slack FJ. MicroRNA therapeutics: towards a new era for the management of cancer and other diseases. Nat Rev Drug Discov. 2017;16(3):203-21.

2. Siegel RL, Miller KD, Jemal A. Cancer statistics, 2017. CA Cancer J Clin. 2017;67(1):7-30.

3. Cronin KA, Lake AJ, Scott S, Sherman RL, Noone AM, Howlader N, Henley SJ, Anderson RN, Firth AU, Ma JM, et al. Annual report to the nation on the status of cancer, part I: National cancer statistics. Cancer. 2018;124(13):2785-800.

4. Bray F, Ferlay J, Soerjomataram I, Siegel RL, Torre LA, Jemal A. Global cancer statistics 2018: GLOBOCAN estimates of incidence and mortality worldwide for 36 cancers in 185 countries. CA Cancer J Clin. 2018;68:394-424.

5. Llovet JM, Zucman-Rossi J, Pikarsky E, Sangro B, Schwartz M, Sherman M, Gores G. Hepatocellular carcinoma. Nat Rev Dis Primers. 2016;2:23.

6. Bertuccio P, Turati F, Carioli G, Rodriguez T, La Vecchia C, Malvezzi M, Negri E. Global trends and predictions in hepatocellular carcinoma mortality. J Hepatol. 2017;67(2):302-9.

7. Ally A, Balasundaram M, Carlsen R, Chuah E, Clarke A, Dhalla N, Holt RA, Jones SJM, Lee D, Ma Y, et al. Comprehensive and integrative genomic characterization of hepatocellular carcinoma. Cell. 2017;169(7):1327-41.

8. Bruix J, Gores GJ, Mazzaferro V. Hepatocellular carcinoma: clinical frontiers and perspectives. Gut. 2014;63(5):844-55.

9. Wong CM, Tsang FHC, Ng IOL. Non-coding RNAs in hepatocellular carcinoma: molecular functions and pathological implications. Nat Rev Gastroenterol Hepatol. 2018;15(3):137-51.

10. Bhan A, Soleimani M, Mandal SS. Long noncoding RNA and cancer: a new paradigm. Cancer Res. 2017;77(15):3965-81.

11. Batista PJ, Chang HY. Long noncoding RNAs: cellular address codes in development and disease. Cell. 2013;152(6):1298-307.

12. Cheetham SW, Gruhl F, Mattick JS, Dinger ME. Long noncoding RNAs and the genetics of cancer. Br J Cancer. 2013;108(12):2419-25.

13. Huarte $M$. The emerging role of IncRNAs in cancer. Nat Med. 2015;21(11):1253-61.

14. Xin XR, Wu MY, Meng QY, Wang C, Lu YA, Yang YX, Li XN, Zheng QD, Pu H, Gui X, et al. Long noncoding RNA HULC accelerates liver cancer by inhibiting PTEN via autophagy cooperation to miR15a. Mol Cancer. 2018;17:16.

15. Jiang RQ, Tang JW, Chen Y, Deng L, Ji J, Xie Y, Wang K, Jia W, Chu WM, Sun BC. The long noncoding RNA Inc-EGFR stimulates T-regulatory cells differentiation thus promoting hepatocellular carcinoma immune evasion. Nat Commun. 2017:8:15.

16. Wang X, Sun W, Shen WF, Xia MY, Chen C, Xiang DM, Ning BF, Cui XL, Li $\mathrm{HY}$, Li XF, et al. Long non-coding RNA DILC regulates liver cancer stem cells via IL-6/STAT3 axis. J Hepatol. 2016;64(6):1283-94.

17. Xin X, Wu M, Meng Q, Wang C, Lu Y, Yang Y, Li X, Zheng Q, Pu H, Gui X, et al. Long noncoding RNA HULC accelerates liver cancer by inhibiting PTEN via autophagy cooperation to miR15a. Mol Cancer. 2018;17:94.

18. Jiang R, Tang J, Chen Y, Deng L, Ji J, Xie Y, Wang K, Jia W, Chu W-M, Sun B. The long noncoding RNA Inc-EGFR stimulates T-regulatory cells differentiation thus promoting hepatocellular carcinoma immune evasion. Nat Commun. 2017:8:15129.

19. Liu RT, Cao JL, Yan CQ, Wang Y, An CJ, Lv HT. Effects of LncRNA-HOST2 on cell proliferation, migration, invasion and apoptosis of human hepatocellular carcinoma cell line SMMC-7721. Biosci Rep. 2017;37:12.

20. Ji J, Yin Y, Ju HY, Xu XL, Liu W, Fu Q, Hu JJ, Zhang XD, Sun BC. Long noncoding RNA Lnc-Tim3 exacerbates CD8 T cell exhaustion via binding to Tim-3 and inducing nuclear translocation of Bat3 in HCC. Cell Death Dis. 2018;9:11.

21. Liu F, Yuan JH, Huang JF, Yang F, Wang TT, Ma JZ, Zhang L, Zhou CC, Wang F, Yu J, et al. Long noncoding RNA FTX inhibits hepatocellular carcinoma proliferation and metastasis by binding MCM2 and miR-374a. Oncogene. 2016;35(41):5422-34.

22. Iorio MV, Croce CM. MicroRNA dysregulation in cancer: diagnostics, monitoring and therapeutics. A comprehensive review. EMBO Mol Med. 2012;4(3):143-59. 
23. Iorio MV, Croce CM. microRNA involvement in human cancer. Carcinogenesis. 2012;33(6):1126-33.

24. Muhammad N, Bhattacharya S, Steele R, Ray RB. Anti-miR-203 suppresses ER-positive breast cancer growth and stemness by targeting SOCS3. Oncotarget. 2016;7(36):58595-605.

25. Anastasiadou E, Jacob LS, Slack FJ. Non-coding RNA networks in cancer. Nat Rev Cancer. 2018:18(1):5-18.

26. Kleaveland B, Shi CY, Stefano J, Bartel DP. A network of noncoding regulatory RNAs acts in the mammalian brain. Cell. 2018;174(2):350-62.

27. Li L, Lv GH, Wang B, Kuang L. The role of IncRNA XIST/miR-211 axis in modulating the proliferation and apoptosis of osteoarthritis chondrocytes through CXCR28 and MAPK signaling. Biochem Biophys Res Commun. 2018;503(4):2555-62.

28. Wang C, Qi S, Xie C, Li C, Wang P, Liu D. Upregulation of long noncoding RNA XIST has anticancer effects on epithelial ovarian cancer cells through inverse downregulation of hsa-miR-214-3p. J Gynecol Oncol. 2018;29(6):e99.

29. Xing F, Liu Y, Wu SY, Wu KR, Sharma S, Mo YY, Feng JM, Sanders S, Jin GX, Singh $\mathrm{R}$, et al. Loss of XIST in breast cancer activates MSN-c-met and reprograms microglia via exosomal miRNA to promote brain metastasis. Cancer Res. 2018;78(15):4316-30.

30. Long J, Yin Y, Guo H, Li S, Sun Y, Zeng C, Zhu W. The mechanisms and clinical significance of PDCD4 in colorectal cancer. Gene. 2018;680:59-64.

31. Wang XJ, Patenode C, Roizman B. U(S)3 protein kinase of HSV-1 cycles between the cytoplasm and nucleus and interacts with programmed cell death protein 4 (PDCD4) to block apoptosis. Proc Natl Acad Sci USA. 2011;108(35):14632-6.

32. Wedeken L, Ohnheiser J, Hirschi B, Wethkamp N, Klempnauer K-H. Association of tumor suppressor protein Pdcd4 with ribosomes is mediated by protein-protein and protein-RNA interactions. Genes Cancer. 2010;1(3):293-301.

\section{Publisher's Note}

Springer Nature remains neutral with regard to jurisdictional claims in published maps and institutional affiliations.
Ready to submit your research? Choose BMC and benefit from:

- fast, convenient online submission

- thorough peer review by experienced researchers in your field

- rapid publication on acceptance

- support for research data, including large and complex data types

- gold Open Access which fosters wider collaboration and increased citations

- maximum visibility for your research: over $100 \mathrm{M}$ website views per year

At BMC, research is always in progress.

Learn more biomedcentral.com/submissions 